\title{
Organización del Trabajo Legislativo y Sistema de Partidos: el Caso del Uruguay
}

\author{
Francisco Sánchez
}

Resumen. En el artículo se sostiene que la estructuración y organización del trabajo legislativo influye y es influida por el sistema de partidos. Se parte del supuesto de que la manera en que se regule el trabajo de los partidos en el Parlamento, servirá como dispositivo para fortalecer o debilitar a los otros arreglos institucionales que intervienen en la formación del sistema de partidos. No se trata de un estímulo inicial -de una variable independiente en sentido puro-, sino más bien de un mecanismo que vigoriza y sostiene los incentivos generados por los otros subsistemas del sistema político. Para demostrar la hipótesis se estudia el caso de Uruguay donde la estructura interna del Parlamento actúa fortaleciendo a las fracciones internas de los partidos caracterizada por posibilitar la competencia en y entre los partidos.

El objetivo de este trabajo es contrastar la hipótesis que sostiene que la estructuración y organización del trabajo legislativo influye en la configuración del sistema de partidos. En el caso concreto del Uruguay, fortaleciendo a las fracciones de los partidos. No se trata de un estímulo inicial -de una variable independiente en sentido puro-, sino más bien de un mecanismo que vigoriza y sostiene los incentivos del sistema electoral uruguayo, caracterizado por posibilitar la competencia en y entre los partidos. Pretendo aportar evidencia acerca del ascendiente de los Legislativos en la morfología de los sistemas de partidos. Parto del supuesto de que, la manera en que se regule su trabajo en el parlamento, servirá como dispositivo para fortalecer o debilitar a los otros arreglos institucionales que intervienen en la formación del sistema de partidos.

He escogido el caso de Uruguay porque la peculiar estructura fraccionada de los partidos y su sistema electoral hacen más evidente el vínculo que pretendo estudiar. Por otro lado, se trata de un sistema de partidos y de una democracia estables. La mayoría de trabajos sobre los partidos políticos uruguayos se pregunta en algún momento sobre el estatuto teórico de las organizaciones políticas de ese país . Casi todos los cuestionamientos se formulan en torno a la relación sui generis entre las fracciones y las matrices partidistas. Los argumentos van desde considerar a los partidos como identidades políticas y sociales, antes que organizaciones estructuradas, hasta calificarlos más bien como alianzas o coaliciones de grupos políticos diversos que comparten algún tipo de afinidad o se asocian en la búsqueda del poder.

La particular estructura del sistema de partidos uruguayo ha sido considerablemente estudiada ${ }^{2}-\mathrm{si}$ se compara con otros países de América Latina- y muchos de esos trabajos resaltan la importancia del sistema electoral, la llamada Ley de Lemas, como un potente incentivo institucional en su articulación, desarrollando así el debate clásico que tiene como punto de referencia las llamadas Leyes de Duverger.

Sin embargo, en el estudio de los estímulos institucionales que dan forma al sistema de partidos, no se ha profundizado sobre el papel de una serie de arreglos institucionales que potencian los incentivos generados por el sistema electoral, contribuyendo a mantener y fortalecer la estructura de competencia partidista.

Comenzaré mostrando las conexiones entre sistema electoral y sistema de partidos, centrándome en la discusión generada en torno al caso uruguayo, para, 
a continuación, señalar algunos aportes teóricos que relacionan los sistemas de partidos con la estructura del trabajo legislativo. En la parte empírica analizaré, desde el punto de vista formal y político, a los sectores parlamentarios y a las comisiones.

\section{Los arreglos institucionales y la morfología del sistema de partidos: sistema electoral y organización del trabajo legislativo}

A pesar de diferencias, las explicaciones sobre la forma del sistema de partidos uruguayo recurren, en algún momento, a mostrar el impacto del sistema electoral sobre la "fraccionalización" de los partidos ${ }^{3}$. Las posiciones se podrían agregar en dos grandes grupos: en el primero estarían las que plantean que el fraccionamiento de los partidos responde más a las peculiaridades del proceso histórico-político uruguayo y limitan la influencia del sistema electoral al hecho de que permite la coexistencia de diversos grupos dentro de los partidos. Para estos autores, el influjo del sistema electoral no radicaría en el incentivo a la creación de fracciones, sino en no limitar las posibilidades de desarrollo de las existentes. Esta lectura más historicista del fraccionamiento era habitual en los primeros trabajos que trataron el tema (Real de Azúa, 1971, 1984; Solari, 1991).

El otro grupo de explicaciones pone más énfasis en el sistema electoral al considerarlo una de las causas principales de la "fraccionalización". El incentivo estaría en que genera competencia interna dentro de las organizaciones políticas a través del sistema de doble voto simultáneo ${ }^{4}$, mecanismo que, en un contexto de elecciones generales, permite al votante realizar una especie de selección interna dentro de los partidos, como si de elecciones primarias se tratase. Botinelli (1990) va más allá y dice que los electores uruguayos seleccionan primero un partido, dentro del partido a las fracciones que compiten, $y$, por último y de darse el caso, a un candidato dentro de la misma fracción ${ }^{5}$.

La discusión clave, más allá de la relación entre sistema electoral y el proceso de separación en fracciones, que ya casi nadie discute sino más bien se posiciona respecto al grado de determinación que éste tiene en el fraccionamiento del sistema de partidos, es clarificar las características de las fracciones 0 , dicho de otro modo, establecer a qué se puede llamar fracciones. Buquet (2000; 1998) argumenta a favor de centrar el análisis en las fracciones de carácter nacional y evitar dar demasiada importancia a las departamentales, ya que éstas, por lo general, se adscriben a uno de los grupos nacionales, y ello en parte por la misma lógica del sistema electoral que combina candidatos de distrito departamental con los nacionales ${ }^{6}$. Las fracciones relevantes, las que cuentan en el proceso de toma de decisiones, serían aquellas que suponen un corte vertical en el partido entero.
No quiero finalizar este apartado sin señalar que no sólo las fracciones son relevantes dentro del sistema político uruguayo, los partidos como unidad también cumplen funciones muy asociadas al particular desarrollo histórico-político del país que le han dotado de características propias. La explicación más común es que los partidos han trascendido el plano de lo político para convertirse en identidades sociales. Abundando en la idea, los políticos uruguayos, aunque se formen en el seno de fracciones en competencia, tienen en común ciertas identidades compartidas a través de las que se reconocen como miembros de un partido. De otra manera, no se podría explicar porqué se mantienen dentro del Lema en lugar de formar un partido propio. Además, el control y mantenerse dentro de los partidos permite a las fracciones el acceso a una serie de recursos a los que por sí solas no podrían optar, sirvan como ejemplo, los cargos en las altas estructuras del Estado.

Es importante tener en cuenta que no conviene asimilar a los sublemas electorales con las fracciones, pues habitualmente se correspondían a las candidaturas presidenciales y en la actualidad más bien a las del Senado, sin embargo, se dan casos de alianzas internas dentro de los partidos para promocionar un candidato presidencial o listas senatoriales. Otro criterio, para ubicar las fracciones dentro de los partidos en el momento postelectoral, es a través de los "sectores parlamentarios" en el interior de las cámaras ${ }^{8}$. Una aproximación desde este punto puede ser el trabajo de Morgenstern (2001) donde se demuestra, empíricamente, que los diputados electos bajo las listas de un determinado Senador votarán en la Cámara de Representantes en el mismo sentido que ese Senador.

Una de las líneas de investigación mas frecuente sobre la relación entre partidos y estructura de la organización del legislativo, ha sido la que explica la disciplina partidista como debida, en parte, al control de los partidos sobre los legisladores. Y ello gracias al manejo que ejercen sus cúpulas sobre algunos aspectos centrales de la estructura del legislativo $y$, por extensión, de la estructura de la toma de decisiones y de las oportunidades políticas. (Cox y Mc Cubbins, 1993; Figueiredo Cheibud y Limongi, 1999; Morgenstern y Nacif, 2002; Carey, 2006).

Pero la manera en que se configura y reparte el trabajo de las cámaras no sólo media en la relación entre diputados y partidos, sino que puede alterar la correlación de fuerzas del propio sistema de partidos. Para explicar las razones de la influencia de la organización en las relaciones de poder, Strøm (1995) plantea que cualquier ordenación dentro del Legislativo crea "grupos de privilegio", los mismos que generan desigualdades en el acceso y control de los recursos políticos, económicos y humanos de las cámaras, a la vez que establecen cierta jerarquía entre los parlamentarios ya que, si bien todos los legisladores son electos iguales, toda regla de 
organización viola esa equidad al dotar, a algunos de ellos y a sus grupos, de mayores capacidades sobre otros. Dependiendo de cómo se distribuyan las plazas en el seno del Congreso, puede darse una suerte de remodelación de fuerzas políticas gracias a la penalización en cargos que sufran los partidos que estén fuera de la mayoría y viceversa.

Pero en este trabajo no me planteo solamente analizar la forma en que la organización de las legislaturas fortalece a las fracciones, como lo hicieron Brady y Bullock (1983), ni trato de examinar la "organización industrial" (Weingast y Marshall, 1988) del Legislativo Uruguayo o la forma en que las "Legislative Rules" (Cox, 2000) influyen en los resultados del proceso legislativo. Se trata más bien de mostrar que los mecanismos de organización de los Legislativos son parte del entramado institucional que influye en la estructura de competencia partidista.

En este punto, me aproximo a la tesis de Colomer (2003) que señala que los sistemas electorales no son la variable que configura los sistemas de partidos, sino que más bien éstos se han ido adaptando a las formas que iba tomando la competencia partidaria. Desde esa óptica, los arreglos institucionales sirven para estabilizar la estructura del sistema de partidos. Aplicando el argumento al tema de este artículo, se podría decir que las estructuras del trabajo legislativo son parte de los mecanismos institucionales que conforman y sostienen a los sistemas de partidos. Los arreglos eficientes serían los que refuerzan el conjunto del entramado institucional, mientras que los mecanismos que no lo hacen, generarían incentivos negativos al fomentar conductas de los diputados que debiliten a los partidos y sus estructuras de competencia. En el caso que presento a continuación, se podrá ver claramente que la organización de las Cámaras fortalece y refuerza la urdimbre institucional que posibilita la representación política a través de las fracciones partidistas.

\section{Los partidos en el Legislativo: los sectores parlamentarios}

La cristalización del mecanismo institucional a través del cual las fracciones y, por extensión, los partidos controlan el poder legislativo, está en los "sectores parlamentarios". Por tales, se entiende "a toda agrupación que, con Lema propio, hubiera obtenido representación en las cámaras" (art. 146 RCS y art. 18 RCR). Si bien los artículos no hablan específicamente de partidos, lo hacen a través de los llamados Lemas que, con precisiones que no vienen al caso, es la denominación que utiliza la legislación electoral del Uruguay para referirse a los partidos.

Pero, en lo que interesa a este trabajo, los reglamentos contemplan una serie de particularidades para adaptar el procedimiento de creación de sectores a las características del sistema electoral y de partidos uruguayo, construyendo un mecanismo que fortalece los incentivos del sistema electoral, a la vez que a las fracciones, al permitirles actuar con entidad propia en el proceso de toma de decisiones. En este sentido, en los reglamentos se dice también que, siempre y cuando los interesados así lo soliciten al Presidente de sus respectivas cámaras, podrán formar un sector los grupos constituidos por legisladores electos en listas individualizadas por un mismo sublema ${ }^{10}$; los legisladores electos en listas que en el último acto eleccionario hubieran postulado los mismos candidatos para la Cámara de Senadores; así como los legisladores que, aunque electos dentro de un mismo sublema o lista de candidatos al Senado, definan su actuación como agrupación distinta y tengan presencia nacional y autoridades propias reconocidas por las autoridades del lema.

El detalle de causales del párrafo anterior responde a las posibilidades que ofrece el sistema electoral uruguayo para la participación de las distintas fracciones integradas dentro de un mismo partido. El modelo de "sectores parlamentarios" permite la actuación de las fracciones internas de los partidos, aunque éstas sean minoritarias y hayan llegado con un solo miembro. El sistema permite también que los grupos que participaron electoralmente como aliados dentro del partido retomen su autonomía después de la elección. Aunque no siempre coincidirán los mismos sectores en las dos Cámaras, lo más probable es que las fracciones más representativas cuenten con miembros en ambas.

Los sectores parlamentarios tienen un alto nivel de protección jurídica, debido a que su formación y creación están claramente reglamentadas, y sirven a la vez como base de los otros medios de organización del trabajo legislativo, como las comisiones. Las pautas para la formación de los sectores son poco flexibles y están centradas en los partidos políticos y el proceso electoral, lo que eleva la barrera institucional para la formación de sectores "ad hoc" que quieran alterar la estructura del sistema de partidos. Aunque la reglamentación permita una serie de variaciones para la formación de los sectores, los partidos siguen siendo en última instancia el criterio de organización, ya que todo el sistema de sublemas y aprobación de listas depende de las autoridades del lema. Los criterios para la formación de sectores no hacen más que adaptarse a la lógica de funcionamiento del sistema de partidos uruguayo.

Aunque las disposiciones para la formación de sectores parlamentarios limitan las condiciones de pertenencia de los legisladores a los mismos, la práctica política permite cierta flexibilidad para el cambio de sector de los cargos electos, si bien con ciertas particularidades que explico: en caso de haber cambios, éstos no conllevan mudar de lema (partido) sino que, sobre todo, implican movimientos que se producen dentro de los sectores de un mismo lema y responden a la reorganización interna de fuerzas en el propio partido. En caso 
de que una nueva fracción del partido pretenda constituirse en sector parlamentario, el Presidente de la Cámara suele acordar el beneplácito de los otros sectores del partido, a manera de gesto de respeto a las lógicas internas de los diversos lemas.

En lo que se refiere a los recursos humanos y materiales, éstos se asignan a los sectores y los legisladores, salvando la diferencia de que a los sectores más grandes se les otorgan algunas facilidades más; pero tanto los sectores como los legisladores cuentan con personal de confianza e infraestructura. No hay que olvidar que, en lo que tiene que ver con los servicios y la asesoría, cada una de las Cámaras, y el Parlamento en su conjunto, poseen personal y medios a los que tienen acceso todos los sectores, diputados y senadores.

Respecto al control político y a la elaboración de leyes, el sistema uruguayo está centrado, formalmente, en los representantes y senadores como individuos, antes que en los sectores. Esto no quiere decir que los legisladores que actúan dentro de un sector no se agrupen para dar más fuerza a sus iniciativas, logrando así que quede más clara su posición en los procesos políticos. Además, los sectores parlamentarios son centrales en el proceso de formación de las comisiones legislativas y, por lo tanto, influyen en el proceso legislativo, pero este aspecto lo explicaré más adelante.

Cuadro 1. Resumen de las características formales de los sectores parlamentarios en el Uruguay.

\begin{tabular}{|l|l|}
\hline Indicador & Valor \\
\hline Estatuto jurídico de los bloques legislativos & Alto \\
\hline Pautas para la formación de bloques & $\begin{array}{l}\text { Flexibilidad: Poca } \\
\text { Criterio: Partido }\end{array}$ \\
\hline Normas para cambio entre bloques & Se permite el cambio bajo ciertos causales \\
\hline Recursos a los que accede el sector & $\begin{array}{l}\text { Humanos y materiales punto medio entre recursos a los } \\
\text { bloques y legisladores y recursos a los legisladores }\end{array}$ \\
\hline
\end{tabular}

Se puede afirmar que los partidos uruguayos y sus fracciones tienen un control relativamente alto del proceso legislativo, debido a su estatuto jurídico que les dota de un buen nivel deinstitucionalización formal; mientras que, por el lado de la institucionalización informal, los sectores parlamentarios se benefician del arraigo de la partidocracia en el Uruguay. Cabe pensar que la dotación de recursos humanos, materiales y políticos a los legisladores les podría servir como un incentivo para "resistirse" a las decisiones de los sectores; pero en este caso, debido precisamente a la fuerte institucionalización de los partidos y al control que tienen de la estructura de decisiones del Legislativo, los costos de entrar en juegos de poder con los sectores son mucho más elevados para los diputados que los potenciales beneficios. Por otro lado, la mayoría de los sectores tienen un tamaño bastante reducido y los diputados y senadores son por lo general los líderes o dirigentes de los mismos, aminorando así las posibilidades de enfrentamiento por intereses. En los casos de sectores más grandes, los senadores y diputados se benefician del arraigo electoral y del poder que éstos tienen. En resumen, los partidos tienen una considerable presencia en el proceso legislativo, a pesar de que los diputados siguen gozando de iniciativa individual. La actuación legislativa suele ir encaminada a fortalecer al partido o fracción, a la vez que sirve para construir las carreras políticas de los legisladores.

Una de las principales razones de ser de los grupos, sectores o bloques, es la de dotar a los partidos, como organizaciones, de presencia en los Legislativos; a la vez que implica contar con un mecanismo que les permite controlar, en mayor o menor medida, el desempeño de los legisladores electos bajo sus listas. Consentir que se reproduzca la división en fracciones característica del sistema de partidos en el legislativo, propicia que los sectores actúen de manera diferente en los plenos o comisiones, lo que les garantiza independencia e identidad sin alejarse de la matriz (lema). En este sentido, genera incentivos similares a los del sistema electoral.

Tratar de imponer unidad de acción a los diputados y senadores electos bajo un mismo lema, sería querer ir en contra del sistema de representación política que se ha ido labrando a lo largo de la historia política uruguaya. Este sistema tiene como eje la diversidad interna de los actores y la pluralidad de núcleos de poder dentro de los partidos. Con esto no estoy diciendo que los legisladores electos bajo un mismo lema no actúen unificados, sino que esto sucede mayormente por las negociaciones internas entre las distintas fracciones de los partidos convertidas en unidades de trabajo legislativo ${ }^{11}$.

La construcción de coaliciones pasa por las fracciones antes que por los partidos políticos. A pesar de que los partidos consigan acuerdos de coalición, como es el caso de la que se formó entre los partidos Colorado y Nacional en 2000, en su interior se mantienen fracciones reacias a los acuerdos que no siempre apoyarán las decisiones del gobierno (Lanzaro, et al 2000).

Como consecuencia de las particularidades del sistema de partidos, carece de sentido analizar a 
los sectores parlamentarios como un mecanismo que garantice la unidad de los partidos y evite el transfuguismo. Más bien, planteo que los sectores parlamentarios son instancias organizativas que sirven para fortalecer a las fracciones de los partidos políticos. La primera constatación de lo dicho está en las ya comentadas normas para la formación de los sectores en las dos Cámaras, que incluyen todas las posibilidades de participación electoral que puede tener una fracción. Por otro lado, la constitución como sector parlamentario de una fracción dota a la misma de una serie de recursos humanos, materiales y políticos, a la vez que le permite una presencia constante en la opinión pública durante los ciclos interelectorales. Las fracciones de los partidos políticos uruguayos tienen una necesidad constante de demostrar su línea política y claridad de planteamientos para así definir mejor su espacio dentro de la correlación de fuerzas internas del partido al que pertenecen y de cara al electorado, labor en la que resulta fundamental el Parlamento.

A pesar de que existe bastante independencia de actuación de las fracciones, ello no elimina el riesgo de que se vean acometidas por una estrategia de fuerte captación de sus integrantes por parte del gobierno, otros sectores u otros partidos. Sin embargo, y sin querer negar la posibilidad, hay algunas instituciones formales e informales que generan un marco de oportunidades con demasiados costos para los políticos que infrinjan las reglas. El uso de la estrategia de buscar apoyo entre los legisladores como individuos, le traería al gobierno más costos que beneficios. En primer lugar, se romperían las pautas de cooperación entre los partidos que se han ido formando a través de décadas en base a la confianza. Además, los partidos o fracciones afectadas podrían actuar a la defensiva y entrar en una línea de fuerte oposición al gobierno, metiéndose así éste en un problema peor que el que buscaba solucionar a través de la captación de los legisladores. Por otro lado, al tratarse de un sistema bicameral, tendría que buscar apoyos individuales en las dos Cámaras, por lo que los efectos negativos de su acción prácticamente se duplicarían ${ }^{12}$. Por último, no hay que olvidar que los partidos controlan de manera centralizada la estructura de oportunidades de las Cámaras, y pueden sancionar y presionar a un legislador que se haya salido de los "parámetros establecidos" como una forma de sentar un precedente y desincentivar a quienes quieran imitarlo.

Para complementar la idea anterior, quiero insistir en un asunto que ya indiqué brevemente: me refiero a que los sectores parlamentarios son por lo general pequeños y sus miembros suelen ser a la vez los dirigentes, líderes y mentores de las fracciones, por lo que es muy difícil que las abandonen para integrarse en otras fracciones o sectores. En el caso de los sectores más grandes de los partidos, es más probable que se formen grupos de legisladores de primera y segunda línea. Esto, de cierta forma, podría incentivar a algún diputado o senador a que abandonase el sector y se integrara en otro en el que tuviese mayor protagonismo; pero, aún en este caso, los beneficios electorales o de acceso a recursos que tiene, siendo parte de una gran fracción, pueden ser mucho mayores. A pesar de las salvedades, hay cambios de políticos y fracciones entre partidos que se han dado en circunstancias específicas que explico más abajo.

Observo gran correspondencia entre las fracciones de los partidos y los sublemas electorales, que casi siempre se componen de candidaturas al Senado y a la Cámara de Representantes. Una vez asignados los escaños, lo más corriente es que casi todas las fracciones que obtuvieron representación en el Senado logren también escaños en la cámara baja, aunque en la Cámara de Representantes es muy común la presencia de un número mayor de fracciones gracias a una serie de alianzas que se forman durante las elecciones, pero que se rompen una vez alcanzados los escaños.

En el cuadro 2 se pueden ver los sectores parlamentarios del Senado, que también estaban presentes en la Cámara de Representantes, durante las dos legislaturas estudiadas. Las mayores variaciones corresponden al Partido Nacional en el que, junto a las fracciones tradicionales más antiguas, Herrerismo y Movimiento Nacional de Rocha, aparecen las nuevas fracciones: Alianza Nacional y Correntada Wilsonista, lideradas por dos jóvenes senadores blancos. Por otro lado, desapareció del Senado, aunque mantuvo un diputado, la fracción Propuesta Nacional, lo que se debió a la combinación de una serie de factores como la pérdida de representatividad de la fracción dentro del partido, ocasionada a su vez por la cuestionable gestión de su líder cuando ejerció la presidencia del directorio del Partido Nacional, así como por la negativa del mismo de encabezar las listas para el Senado. Mientras las fracciones del Frente Amplio se mantuvieron estables, las del Partido Colorado estuvieron nucleadas en torno a la Lista 15, liderada por el ex Presidente Jorge Batlle y el Foro Batllista, liderada por el ex Presidente Julio Sanguinetti. Ésta última fracción sirvió también como una especie de coalición entre pequeñas fracciones del Partido Colorado, que habían ido perdiendo su espacio debido a los cambios en el electorado o a la desaparición de sus dirigentes más importantes. En este caso, estarían la fracción llamada Agrupación por el Gobierno del Pueblo - Lista 99, que perdió importancia en la política uruguaya, en comparación con el tiempo en que estaba liderada por Zelmar Michelini o por el ex Vicepresidente Hugo Batalla. Un poco más incierta era la situación de fracciones pequeñas como Cruzada 94, liderada por el entonces senador Pablo Millor que también fue elegido dentro de la lista del Foro, pero que todavía contaba con políticos identificados con ella en las dos Cámaras. 
Cuadro: 2. Sectores parlamentarios del Senado de Uruguay.

\begin{tabular}{|c|c|c|c|c|}
\hline \multirow[b]{2}{*}{$\begin{array}{l}\text { Partidos } \\
\text { (Lemas) }\end{array}$} & \multicolumn{2}{|l|}{1995} & \multicolumn{2}{|c|}{2000} \\
\hline & Sector & $\mathbf{N}^{\circ}$ de Senadores & Sector & $\mathbf{N}^{\circ}$ de Senadores \\
\hline \multirow[t]{5}{*}{$\begin{array}{l}\text { Partido } \\
\text { Nacional }\end{array}$} & $\begin{array}{r}\text { Movimiento Nacional } \\
\text { de Rocha }\end{array}$ & 1 & $\begin{array}{r}\text { Movimiento } \\
\text { Nacional de Rocha }\end{array}$ & 1 \\
\hline & Herrerismo & 4 & Herrerismo & 4 \\
\hline & Propuesta Nacional & 2 & --- & \\
\hline & -- & -- & Alianza Nacional & 1 \\
\hline & -- & -- & Correntada Wilsonista & 1 \\
\hline \multirow[t]{6}{*}{ Frente Amplio } & $\begin{array}{l}\text { Movimiento de } \\
\text { Participación Popular }\end{array}$ & 1 & $\begin{array}{r}\text { Movimiento de } \\
\text { Participación Popular }\end{array}$ & 2 \\
\hline & Democracia Avanzada & 1 & $\begin{array}{r}\text { Democracia } \\
\text { Avanzada-1001 EP-FA }\end{array}$ & 1 \\
\hline & Vertiente Artiguista & 1 & Vertiente Artiguista & 2 \\
\hline & Asamblea Uruguay & 4 & Asamblea Uruguay & 2 \\
\hline & $\begin{array}{r}\text { Unidad y Pluralismo } \\
\text { Frenteamplista, } \\
\text { Lista } 90\end{array}$ & 2 & Partido Socialista & 4 \\
\hline & Alianza Progresista & 1 & Alianza Progresista & 1 \\
\hline \multirow{4}{*}{$\begin{array}{l}\text { Partido } \\
\text { Colorado }\end{array}$} & batllismo, Lista 15 & 1 & batllismo, Lista 15 & 5 \\
\hline & --- & --- & $\begin{array}{l}\text { Agrupación por } \\
\text { el Gobierno del } \\
\text { Pueblo-Lista } 99\end{array}$ & 1 \\
\hline & Foro Batllista (Acuerdo) & & Foro Batllista & 5 \\
\hline & Cruzada 94 & 2 & ¿? & -- \\
\hline Nuevo Espacio & Nuevo Espacio & 1 & Nuevo Espacio & 1 \\
\hline
\end{tabular}

Fuente: Elaboración propia a partir de datos del Archivo Parlamentario de Uruguay

En la Cámara de Representantes había sectores que no tenían presencia nacional como para alcanzar un escaño en el Senado. En el Partido Nacional estaba la Línea Nacional de Florida, mientras que en el Frente Amplio estaba la Unión Frenteamplista, que se formó a partir de una crisis del Partido Comunista de Uruguay, y la fracción 26 de marzo del diputado Raúl Sendic.

Los cambios de políticos entre los distintos partidos son hechos aislados y responden a coyunturas de crisis 0 a enfrentamientos más o menos estructurales dentro de las formaciones de origen, más que a simples juegos de conveniencia clientelar respecto al gobierno. No he podido encontrar el caso de un político, en activo, que haya transfugado entre los partidos tradicionales, mientras que es muy común que el Frente Amplio sea el receptor de las deserciones de los otros partidos. Este comportamiento ya se notó desde la fundación del Frente en la que estuvieron los sectores más de izquierda de los partidos tradicionales. Desde la transición hasta ahora, el Frente ha seguido acogiendo políticos disidentes. Los casos más notorios son los de Rodolfo Nin Noboa, exintendente y senador electo que abandonó el Partido Nacional aduciendo discrepancias con la línea del partido bajo la dirección del expresidente Lacalle, que llegó a la vicepresidencia del Uruguay en las listas del Frente. Su incorporación se dio en el marco del llamado Encuentro Progresista. Una cosa parecida está sucediendo con el Nuevo Espacio: luego de una división interna, la fracción liderada por el senador Michelini se aproximó al Frente Amplio con miras a las elecciones de 2004, en las que participarían todos juntos bajo el nombre de Nueva Mayoría. Un proceso inverso sucedió con el Partido Para el Gobierno del Pueblo, que comenzó siendo la fracción socialdemócrata del Partido Colorado, del que se separó para integrarse al Frente. Lo que queda de ella ha vuelto a actuar con el Partido Colorado.

Como se puede constatar, las fracciones partidistas en tanto eje del sistema de partidos uruguayo, tienen cabida en la organización del trabajo legislativo, la misma que las mantiene y fortalece. De esta manera, los efectos producidos por otras instituciones, como el sistema electoral, se vigorizan y refuerzan. Cabe recordar que el punto de partida para la formación de los sectores parlamentarios son las mismas listas electorales. 


\section{Partidos y comisiones en el Uruguay}

Una de las funciones principales de los sectores parlamentarios es la de servir como base para la asignación de las comisiones legislativas permanentes en la Cámara de Diputados y en la de Senadores, atribución que dota a las fracciones de forma indirecta del control de los procesos parlamentarios. El vínculo entre comisiones y organizaciones políticas tiene algunas implicaciones para la estrategia de los partidos en el Legislativo, así como también para las relaciones entre poderes, especialmente, si se toma en cuenta que en las comisiones es donde se ordenan las prioridades de la agenda política, se analizan en profundidad las características de los proyectos, se negocian los apoyos partidistas y se hacen las modificaciones necesarias a los proyectos de ley (Lanzaro. (Cood). 2001:84).

La base del reparto de las comisiones son, efectivamente, las negociaciones entre los sectores parlamentarios y los partidos. La cuestión de fondo durante las mismas es conjugar la proporcionalidad de la distribución política de las Cámaras con los intereses de los partidos, fracciones y legisladores. Al parecer, las negociaciones son un poco más complejas en la Cámara de Representantes porque cada diputado sólo puede estar en una comisión y los distintos sectores tratan de colocar a sus diputados en las comisiones más relevantes. El incentivo para evitar posibles bloqueos y para que los sectores partan de una posición de negociación de medianos y no máximos beneficios, está en que la norma fija que las decisiones serán tomadas proporcionalmente de acuerdo con el tamaño de los sectores en las Cámaras. Esto da capacidad de veto a los sectores mayoritarios, aunque por sí solos tampoco podrán tomar decisiones. Al estar la Cámara bastante fragmentada, es más probable llegar a puntos medios de acuerdo antes que a la imposición de un sector. Las negociaciones en el Senado suelen ser más fluidas, porque hay menos legisladores que ubicar y más de un puesto en comisión para cada senador, lo que permite tener un mejor sistema de compensaciones.

El sistema de comisiones de la Cámara de Representantes y Senadores es muy similar en lo que tiene que ver con los tipos y sus competencias, pero hay pequeñas diferencias en la manera en que se integran, la misma que está diseñada para favorecer a los partidos. Las comisiones legislativas como parte del entramado legislativo aparecen en la Constitución uruguaya y, aunque no se dice explícitamente que las Cámaras organizan su trabajo a través de comisiones, se las menciona de forma indirecta ${ }^{13}$. Por otro lado, las comisiones de investigación tienen estatus constitucional gracias a que en el art. 120 de la CPROU se dice que: "las Cámaras podrán nombrar comisiones parlamentarias de investigación o para suministrar datos con fines legislativos".

Las comisiones legislativas de los dos Cámaras se clasifican en dos grandes grupos: las permanentes y las especiales (art. 114,116 RCR y 129,131RCS). Las comisiones permanentes se designan para todo el período y su número y campos de acción están descritos en los respectivos reglamentos de las Cámaras ${ }^{14}$. Las comisiones permanentes pueden actuar de forma integrada cuando se tiene que tratar algún asunto en el que se incluyan temas que correspondan a lajurisdicción de más de una comisión. Las comisiones especiales se eligen para un cometido fijo con el fin de informar sobre asuntos o problemas determinados. Estas comisiones cesarán luego de haber resuelto el asunto que motivó el informe o cuando la propia Cámara así lo resuelva. En el acto legislativo en el que se crea la comisión especial, se fija también el plazo en el que deberá presentar su dictamen, plazo que es prorrogable. Las comisiones investigadoras, aquellas que constan en el art. 120 de la Constitución, se habilitan previo informe de una comisión preinvestigadora compuesta de tres miembros, que dictamina si las causas denunciadas por el Representante o Senador, dan lugar a una investigación por irregularidades (art. 117. art. 118.RCR art. 135 y ss RCS).

Cuadro 3. Tipos de comisiones del Senado y Cámara de Representantes de Uruguay.

\begin{tabular}{|c|}
\hline Tipos de comisiones \\
\hline $\begin{array}{c}\text { Comisiones permanentes } \\
\text { (pueden actuar integradas) }\end{array}$ \\
\hline Comisiones especiales \\
\hline Comisiones de Investigación \\
\hline
\end{tabular}

Para integrar las comisiones permanentes de la Cámara de Representantes se nombra una comisión especial integrada por un delegado de cada sector parlamentario que cuenta con voto proporcional de acuerdo a la representación alcanzada en la Cámara. Ésta determina el número de miembros de cada comisión permanente, número que no puede ser superior a quince ni inferior a tres. El total de miembros de las comisiones es el mismo que el de componentes de la Cámara menos uno (98), para dar así la posibilidad a todos los representantes de que formen parte de una comisión permanente. El Presidente designa, a propuesta de los diferentes sectores, a los representantes que se incorporarán a las comisiones. Los puestos en comisión pertenecen a los sectores parlamentarios $y$, si desean hacer cambios durante la Legislatura, tienen que comunicarlos por escrito al Presidente de la Cámara. Si las modificaciones que se quieren hacer responden a un acuerdo entre los distintos sectores y las mismas afectan la distribución de cargos establecida al iniciarse la Legislatura, la comunicación correspondiente es distribuida por la Secretaría y leída en la primera sesión que se realice para que sea aprobada por el pleno. En caso de que uno o varios de los sectores parlamentarios se considerasen perjudicados por dichos acuerdos, 
lo expresarán así en las sesiones del pleno y se nombrará una nueva comisión especial para buscar un nuevo consenso (art. 15- 17.-RCR).

Las comisiones permanentes del Senado tienen entre cinco y nueve miembros y las especiales e investigadoras se integran según el número que se establezca al disponerse su creación. A pesar de que el reglamento (art. 141 RCS) establece que el número de miembros de las comisiones permanentes lo fija el Senado, a propuesta de la Presidencia, en las actas de las sesiones en las que se acuerda dicho número, tanto el Presidente, como los representantes de los distintos sectores que intervienen en la sesión, hacen mención a una serie de acuerdos previos entre partidos para fijarlo, así como para la distribución dentro de las mismas comisiones. El reparto de los cargos de los sectores parlamentarios en cada una de las comisiones permanentes lo realiza el Presidente del Senado, procurando, en lo posible, mantener en cada comisión y entre los distintos sectores la proporción que éstos tienen en el Senado, así como también la representación en las comisiones del mayor número de sectores. Una vez que el Presidente informa a los sectores del número de integrantes que les corresponde en cada comisión, éstos se encargan de designar a los Senadores ( art. 141-144 RCS). Como se podrá ver, el mecanismo de nombramiento de los miembros de las comisiones permite, a la vez que refleja y mantiene, la estructura del sistema de partidos.

Debido a la diversidad de sectores políticos y a que no todos pueden tener presencia en todas las comisiones, en la Cámara de Representantes también existe la figura de los delegados de sector, que son diputados acreditados en las distintas comisiones con voz pero sin voto. Algo parecido también está contemplado en el Reglamento de la Cámara de Senadores, pero la presencia de estos delegados es menor, ya que hay menos sectores y los senadores suelen ocupar espacios en las comisiones en las que tienen intereses.
La creación de las comisiones permanentes en el Uruguay es muy restringida, ya que su número y competencia está fijado por los respectivos reglamentos de las Cámaras. La presencia de los partidos en las comisiones se fortalece gracias al método de asignación de los miembros de las comisiones, pues no sólo los puestos en comisiones son asignados a los sectores, sino que también tienen la posibilidad de cambiar a los diputados 0 Senadores que los representan.

Las competencias de las comisiones uruguayas a nivel de legislación y control son muy elevadas en las dos Cámaras. En lo que tiene que ver con el proceso de legislación, al tratarse de un bicameralismo simétrico, en el que tanto el Senado como la Cámara de Representantes tienen las mismas atribuciones legislativas y sirven como Cámara de origen o revisora, indistintamente, las potestades de las comisiones son las mismas. Las comisiones de cada Cámara son las encargadas de compatibilizar el proyecto de ley original o aprobado por la Cámara de origen, con las observaciones de los distintos partidos y legisladores en caso de que las haya.

La duración del período de las comisiones permanentes es el mismo que el de toda la legislatura, aunque puede haber variaciones en la composición en caso de que se llegue a nuevos acuerdos entre los sectores parlamentarios. Esto último es poco habitual en el Senado y más común en la Cámara de Representantes, donde la correlación de fuerzas en las comisiones es más ajustada y puede alterarse muy fácilmente. Una de las coyunturas en que esto puede suceder es la elección de Presidente de la Cámara, que se da cada año y va rotando entre los partidos. Es de suponer que el diputado que sea electo Presidente, será uno de los diputados de primera línea y su salida de la comisión en la que actúa para ejercer la Presidencia puede perjudicar a su partido; pues muy posiblemente éste lo habría destinado a una comisión relevante en la que buscase colocar a otro representante de peso político.

Cuadro 4. Resumen de los atributos de las comisiones legislativas en el Uruguay

\begin{tabular}{|c|c|}
\hline Indicador & Valor \\
\hline Creación de las comisiones & Creación fija \\
\hline $\begin{array}{l}\text { Asignación de los miembros } \\
\text { de las comisiones }\end{array}$ & Puestos en comisiones son asignados a los sectores \\
\hline Competencias de las comisiones & $\begin{array}{l}\text { Legislativas: } \\
\text { Presenta un informe que será discutido en el pleno } \\
\text { Control: } \\
\text { Presentan los informes previos en los procesos } \\
\text { de control (Venias Senado) } \\
\text { Funcionarios tienen que acudir a las comisiones } \\
\text { Pueden solicitar documentos o hacer preguntas } \\
\text { Creación de Comisiones de Investigación }\end{array}$ \\
\hline $\begin{array}{l}\text { Duración del período de las } \\
\text { comisiones permanentes }\end{array}$ & $\begin{array}{l}\text { Toda la legislatura, posibilidad de reasignación } \\
\text { previo acuerdo de los sectores }\end{array}$ \\
\hline
\end{tabular}


A partir de los datos arriba expuestos, se puede concluir que el modelo uruguayo de comisiones funciona bastante vinculado a los sectores parlamentarios a través de los partidos políticos $y$, más específicamente, a las fracciones que los integran. Los sectores son la base de toda la estructura del trabajo legislativo, lo que sirve para fortalecerlos y mantenerlos. A pesar de ser un modelo centrado en los partidos, el sistema de comisiones no coarta la iniciativa de los diputados y senadores como individuos en los procesos legislativos y de control, aunque la supervisión final de las iniciativas pasa por la estructura centralizada de toma de decisiones de las Cámaras, que a su vez están intervenidas por los partidos. Además, y en última instancia, siempre se podrá apelar al hecho de que el titular del puesto en comisión es el sector y no el legislador.

La parte formal de la constitución de las comisiones legislativas suele quedar registrada en los diarios de las primeras sesiones del Senado y de la Cámara de Representantes: en ellos hay muy pocas intervenciones de legisladores que hagan algún tipo de pronunciamiento en nombre de sus partidos o sectores. Más que protestar por el reparto de las comisiones, el tono de los discursos suele ir más enfocado a dejar constancia de los "sacrificios" que han hecho sus organizaciones en pro de la búsqueda de los consensos. Asimismo, las votaciones del pleno en las que se decide el reparto de las comisiones son por unanimidad, lo que demuestra que una vez que se aceptan los acuerdos, éstos se mantienen y se cumplen.

Parece ser que los legisladores uruguayos usan las comisiones más como espacio de consenso y debate reposado que como un espacio de presión y chantaje político al gobierno y a los otros partidos. El discurso político y las demandas se dejan para el pleno. Esta actitud es consecuente con la constante necesidad que tienen los sectores de los partidos políticos uruguayos de marcar entre ellos sus posiciones y diferencias de forma clara ${ }^{15}$.

Por último, cabe señalar que contribuye a un mejor desempeño del sistema la estabilidad de las comisiones permanentes, que han tenido muy pocas variaciones desde la transición hasta la actualidad ${ }^{16}$. A pesar de que el sistema de comisiones de la Cámara de Representantes tiene una comisión más que en el Senado, se ha mantenido al máximo la simetría de las jurisdicciones de las comisiones, hecho que a la larga repercute también en el tratamiento de las leyes al crear las respectivas contrapartes en las diferentes Cámaras.

\section{Conclusión}

Como se ha demostrado a lo largo del artículo, la presencia de los partidos políticos, bien a través de sus fracciones, bien de manera unitaria, se deja ver a lo largo de todos los procesos y de la estructura interna del legislativo uruguayo, procesos que se han articulado de tal manera que sirven a su vez para reforzar y reproducir la estructura del propio sistema de partidos de este país. Las fracciones de los partidos, en tanto unidades relevantes de la competencia política en el Uruguay, consiguen mantenerse y reproducirse gracias al papel que les otorga el Parlamento a través de los sectores parlamentarios: eje de todo el trabajo legislativo. Es importante resaltar que la dinámica del trabajo parlamentario refuerza los incentivos generados por otras instituciones, de forma especial por el sistema electoral.

Por último, cabe destacar que los representantes electos tienen capacidad de maniobra como individuos, pero prefieren mantenerse dentro del sistema debido a que la fuerte institucionalización de la partidocracia uruguaya penaliza con elevados costes a quien actúa al margen de los partidos 0 rompe con ellos. Tal es así que resulta más rentable actuar respetando las reglas de juego. 
1 De forma ilustrativa cito el argumento de Sartori (1999: 102), afirmaba que Uruguay (antes de 1973, la aclaración es mía) era un caso dudoso de bipartidismo y que, en realidad, era sólo una fachada de los actores reales del sistema de partidos que para él eran las fracciones (sublemas) de los partidos blanco y colorado. Una argumentación interesante sobre la condición de los partidos uruguayos puede encontrarse en González (1993: 31 y ss.), que reproduce parte de los argumentos más críticos, sobre todo los de Lindahl (1962), para después debatirlos y refutarlos. Una revisión actual de las distintas posiciones analíticas sobre el fenómeno de las fracciones en Uruguay se puede encontrar en Monestier (1999).

2 Sin pretender hacer una lista exhaustiva, cabe citar como referencia los siguientes estudios publicados por investigadores de otros países: Alcántara y Crespo (1992), Sartori (1999), Crespo (2002), Martínez Barahona (2001), Morgenstern (2001), Taylor (1960), Sartori (1999) entre otros. Pero resulta más considerable el trabajo realizado por los académicos locales. Insisto, sin ánimos de hacer una lista exhaustiva, cito aquí como referencia los trabajos de Altman (2000), Bodemer y Laurnaga (1993), Buquet, Chasquetti, y Moraes (1998), Caetano, Rilla, y Peréz (1987), González (1993), González, Monestier, Queirolo, y Sotelo Rico (1999), Lanzaro (2000), Real de Azúa (1971), Solari (1991).

3 González (1993: 44 y ss) resume en tres las posiciones sobre el fraccionamiento de los partidos uruguayos, a las que llama: "optimista", "pesimista" y "la combinada". La primera, más común en el ambiente político y periodístico, rescata la virtud histórica de los partidos convertidos en identidades socio-políticas de adaptarse dinámicamente a las distintas preocupaciones de la sociedad, lo que hacía necesario altos niveles de fraccionamiento, solamente posibles debido a una democracia interna muy desarrollada. En este caso el sistema electoral es una herramienta que permite el mantenimiento de la diversidad. La visión pesimista, más arraigada en los extremos del espectro político y en parte de la intelectualidad, explican las fracciones debido a que el sistema de partidos uruguayo "no está orientado políticamente" sino que responde a un entramado de motivaciones personales y privados en su relación con el aparato del Estado. Es decir, la política uruguaya es vista como un gigantesco sistema de patronazgo. Los partidos serían como cooperativas de empresarios políticos que buscan captar votos. Aparte del clientelismo como factor explicativo, la visión pesimista asigna a la legislación electoral un papel dominante en el mantenimiento del sistema. Como es de esperar, la visión ecléctica del sistema recoge elementos de las dos posiciones y es la más aceptada por los académicos: éstos no niegan el particular origen y desarrollo histórico de los partidos, el clientelismo ni la influencia del sistema electoral, sino que buscan una posición combinada. La diferencia de este sector es que busca hacer una lectura positiva del sistema de partidos a partir del impacto que estos han tenido en el desarrollo de la democracia y el sistema político uruguayo. Por último, quiero resaltar que el trabajo de González se realizó en un momento inicial de la redemocratición, por lo que sus conclusiones no podían incluir los cambios del sistema de partidos en el nuevo escenario social, económico y político. Por otro lado, desde la publicación de su trabajo se han realizado una serie de estudios (Buquet, 2000; Buquet et al., 1998; Monestier, 1999; Morgenstern, 2001) que incluyen un fuerte componente empírico que incorpora nuevos elementos al debate.

4 El sistema electoral uruguayo se caracteriza porque las elecciones generales de Presidente y representantes a las dos cámaras legislativas se realizan cada cinco años y de forma simultánea. Se usa:1) representación proporcional en la elección de ambas cámaras, 2) listas cerradas y bloqueadas, 3) doble voto simultáneo y 4) mayoría simple para decidir quien gana la presidencia, modalidad que rigió hasta 1996, que se pasó al ballotage (González1993:41). Buquet, Chasquetti y Moraes (1998:14) señalan con razón, según mi punto de vista, que es impreciso señalar que las listas uruguayas son cerradas y bloqueadas, concepto que en Ciencia Política está asociado con la opción única del elector y la verticalidad en el control partidista, fenómeno que se limita en el Uruguay gracias al doble voto simultáneo que permite al elector seleccionar entre una serie de listas (posibilidades) para los distintos cargos dentro de un mismo partido. Por otro lado, señalan que el elector hace un voto conjunto (o en bloque) por un paquete de opciones preparado por los distintos sectores de los partidos, en los que se incluye un candidato a la presidencia y candidatos a Senadores y Diputados (la llamada lista).

5 Para Morgenstern (2001:115) el sistema electoral deja también muy claro que las fracciones hacen frente a un contradictorio conjunto de intereses. Por un lado, son incitadas a trabajar por el bien del partido. Por otro, sin embargo, son empujadas a competir unas contra otras en detrimento de la unidad del mismo.

6 El contar las hojas de votación para cuantificar las fracciones (Verrnazza,1989) es el criterio que más críticas ha recibido como forma de cuantificarlas. Monestier $(1999,50)$ cuestiona el enfoque de Buquet (1998) centrado en las grandes fracciones de carácter nacional con el argumento de que no desentraña por completo la lógica con la que operan las divisiones internas de los partidos, sino que reflejaría sólo la epidermis del fenómeno, sólo el resultado de proceso de fraccionamiento. Sin querer dejar de lado este argumento que puede ser válido para otro tipo de investigaciones, aquí comparto el enfoque de Buquet ya que, por la definición de mi objeto de estudio, me interesa comprender los procesos de las grandes fracciones nacionales que son las protagonistas del trabajo parlamentario.

7 Sobre este punto Monestier (1999:43) dice que en un medio político con tradiciones tan arraigadas, las fracciones que abandonan el lema son sancionadas por el electorado a menos de que esto se de en coyunturas históricas críticas que las justifiquen.

8 Los sectores parlamentarios uruguayos son los equivalentes locales a lo que en otros países se conoce como Grupo Parlamentario, Bancada, Bloque, etc.

9 Buquet, Chasquetti y Moraes $(1998,21)$ lo expresan de una mejor manera: “la dinámica política uruguaya tiene su núcleo central en el parlamento porque es allí, en todo caso, donde se producirían los "bloqueos políticos". La composición de las bancadas partidarias, a partir de grupos diferentes y autónomos, es la manifestación concreta del problema que me 
interesa. La fraccionalización partidaria se expresa de forma privilegiada en la necesidad de negociar en el parlamento no con una cabeza por partido sino con una cabeza por sector. Estas últimas, las fracciones de partido, son en general las que cuentan con bancadas parlamentarias absolutamente disciplinadas y, por lo tanto, se transforman en ocasiones en los agentes relevantes en el proceso de toma de decisiones".

10 A partir de las reformas de 1996, esta disposición es solamente válida para la Cámara de Senadores, ya que se prohibieron los sublemas para la Cámara de Representantes.

11 Para una argumentación más extensa sobre las fracciones de los partidos como las verdaderas unidades del trabajo legislativo se puede ver Buquet, Chasquetti, y Moraes (1998).

12 Morgenstern (2001) sostiene que los sectores que representan a las fracciones en las dos Cámaras actúan de forma conjunta y coordinada; para demostrarlo presenta evidencia en el sentido de que los votos de los diputados que se eligieron en la misma papeleta que un determinado senador, casi siempre son en la misma dirección, al igual que los argumentos y posiciones que sostienen en los distintos temas públicos.

13 Entre los artículos de la Constitución uruguaya que hacen mención a las comisiones están: art. 117 “Los Senadores y Representantes serán compensados por sus servicios con una asignación mensual que percibirán durante el término de sus mandatos, sin perjuicio de los descuentos que correspondieran, de acuerdo con el reglamento de la respectiva Cámara, en caso de inasistencias injustificadas a las sesiones de la Cámara que integran o de las comisiones informantes de que forman parte (...)". El art. 180 "Los Ministros podrán asistir a las sesiones de la Asamblea General, de cada Cámara, de la Comisión Permanente y de sus respectivas comisiones internas, y tomar parte en sus deliberaciones, pero no tendrán voto” (...). El Art. 202. “La Enseñanza Pública Superior, Secundaria, Primaria, Normal, Industrial y Artística, serán regidas por uno o más Consejos Directivos Autónomos. Los demás servicios docentes del Estado, también estarán a cargo de Consejos Directivos Autónomos, cuando la ley lo determine por dos tercios de votos del total de componentes de cada Cámara. Los Entes de Enseñanza Pública serán oídos, con fines de asesoramiento, en la elaboración de las leyes relativas a sus servicios, por las Comisiones Parlamentarias (...)" El art. 207 “El Consejo de Economía Nacional se dirigirá a los Poderes Públicos por escrito, pero podrá hacer sostener sus puntos de vista ante las Comisiones Legislativas, por uno o más de sus miembros.(...)".

14 Las comisiones permanentes del Senado en la actualidad son quince: asuntos administrativos; asuntos internacionales; asuntos laborales y seguridad social; ciencia y tecnología; Constitución y legislación; defensa nacional; educación y cultura; ganadería, agricultura y pesca; hacienda; industria y energía; medio ambiente; presupuesto; salud publica, transporte y obras publicas; vivienda y ordenamiento territorial. En la Cámara de Representantes hay 16 comisiones permanentes: asuntos internacionales; asuntos internos; Constitución, códigos, legislación general y administración; defensa nacional; derechos humanos; educación y cultura; ganadería, agricultura y pesca; hacienda; industria, energía y minería; legislación del trabajo; presupuestos; salud publica y asistencia social; seguridad social; transporte, comunicaciones y obras publicas; turismo y vivienda, territorio y medio ambiente.

15 El ex Presidente de la Cámara de Diputados, Stirling (1995) planteaba el tema en los siguientes términos: “a diferencia de otros países en que el trabajo de la comisión es el trabajo por excelencia y el del plenario es simplemente la homologación casi formal del trabajo en comisiones, para nosotros no es así. Aquí se repite todo el proceso argumentativo de las comisiones pero multiplicado, porque hay discursos de tono político porque todos quieren participar y dar su punto de vista". Más adelante pone ejemplos de leyes que tuvieron un tratamiento rápido y consensual en comisión, pero al llegar al pleno el trámite se demora y estanca. Termina diciendo que es muy difícil que se reduzca el rol del plenario en la discusión de las leyes y se fortalezca el papel de las comisiones; para él la razón es: "la propia confrontación que vamos teniendo en los partidos políticos. En Uruguay no tenemos cuatro partidos políticos porque dentro de cada partido hay muchas corrientes. Cada una de estas tiene el legítimo derecho de sentar sus posiciones aún cuando esas posiciones hayan sido perfectamente explicitadas por otros compañeros de su mismo partidos pero no de su misma corriente política".

16 En el Senado y la Cámara de Representantes se crearon sendas comisiones de medio ambiente y de vivienda, mientras que sólo en el Senado hay una comisión de ciencia y tecnología y en Diputados una, de derechos humanos. 


\section{Referencias}

Alcántara, Manuel, and Ismael Crespo. 1992. Partidos políticos y electorales en Uruguay (1971-1990). Madrid: CEDEAL.

Altman, David.2000. "The Politics of Coalition Formation and Survival in Multiparty Presidential Democracies". Party Politics 6(3): 259-283.

Bodemer, Klaus y Maria Elena Laurnaga, eds. 1993. Estructura y funcionamiento de los partidos políticos: una reforma posible. Montevideo: Fesur.

Bottinelli, Oscar. 1990. El sistema electoral uruguayo. Descripción y análisis. Heideberg: WP Universidad de Heideberg.

Brady, David, W., and Charles S. Bullock III. 1983. "Party and Fraccional Organization in Legislatures." Legislative Studies Quarterly 8(4):599-654.

Buquet, Daniel. 2000. “Fragmentación y fraccionalización política: de la oferta electoral a la conformación del Gobierno" In La "segunda" transición en el Uruguay, ed. Jorge Lanzaro. Montevideo: ICP-Fundación de Cultura Universitaria.

Buquet, Daniel, Daniel Chasquetti and Andrés Moraes. 1998. Fragmentación política y gobierno en Uruguay ¿Un enfermo imaginario?. Montevideo: ICP-Universidad de la República.

Carey, John. 2006. “Legislative Organization” In The Oxford Handbook of Political Institutions, eds. Rhodes, Binder and Rockman. Oxford: Oxford University Press.

Caetano, Gerardo, José Rilla y Romeo Peréz. 1987. “La partidocracia uruguaya. Historia y teoría de la centralidad de los partidos políticos". Cuadernos del CLAEH (44)

Colomer, Josep M. 2003. “Son los partidos los que eligen los sistemas electorales (o las leyes de Duverger cabeza abajo)." Revista española de ciencia política 9:39-63.

Cox, Gary. 2000. “On the Effects of Legislative Rules." Legislative Studies Quarterly. 25(2):169-192.

Cox, Gary, and Mathew Mccubbins. 1993. Legislative leviathan: party government in the House. Berkeley, Berkeley: University of California Press.

Crespo, Ismael. 2002. Tres décadas de política uruguaya. Madrid: CIS-Siglo XXI.

Figueiredo, Argelina, and Fernando Limongi. 1999. Executivo e legislativo na nova ordem constitucional. Rio de Janeiro: FGV.

González, Eduardo. 1993. Estructuras políticas y democracias en Uruguay. Montevideo: Fundación de Cultura Universitaria.

González, Jorge, Felipe Monestier, Rosario Queirolo and Mariana Sotelo Rico.1999. Los partidos políticos uruguayos en tiempos de cambio. Montevideo: Fondo de Cultura Universitaria.

Lanzaro, Jorge. ed. 2000. La "segunda" transición en el Uruguay. Montevideo: ICP- Fundación de Cultura Universitaria.

Lindahl, Goran.1962. Uruguay's New Path: A Study in Politics During the First Colegiado, 1919-33. Stockholm: Broderna Lagerstrom.

Martínez Barahona, Elena. 2001. “Uruguay". In Partidos Políticos de América Latina , eds. Manuel Alcántara and Flavia Freidenberg. Salamanca: Ediciones Universidad de Salamanca.

Monestier, Felipe. 1999. “Partidos por dentro: la fraccionalización de los partidos políticos en el Uruguay (19541994)." In Los partidos políticos uruguayos en tiempos de cambio, Eduardo González et al. Montevideo: Fundación de Cultura Universitaria.

Morgenstern, Scott. 2001. “Grupos Organizados y Partidos Desorganizados: Incentivos Electorales en Uruguay". América Latina Hoy.

Morgenstern, Scott and Benito Nacif, eds. 2002. Legislative Politics in Latin America. New York: CUP.

Real De Azúa, Carlos. 1971. "Política, poder y partidos en el Uruguay de hoy". In Uruguay Hoy, ed. Luis Carlos Benvenuto. Buenos Aires: Siglo XXI.

Real De Azúa. Carlos. 1984. Uruguay, ¿Una sociedad amortiguadora? Montevideo: Ediciones de la Banda Oriental.

Sartori, Giovanni. 1999. Partidos y sistemas de partidos. Madrid: Alianza.

Solari, Aldo. 1991. Partidos políticos y sistema electoral. Montevideo: Fundación de Cultura Universitaria.

Strom, Kaare. 1995. "Parliamentary Government and Legislative Organisation". In Parliaments and Majority Rule in Western Europe, ed. Herbert Döring. Frankfurt: Campus Verlag.

Taylor, Philip. 1960. Governments and politics of Uruguay. New Orleans: Tulane University.

Vernazza, Francisco. 1989. "Minoristas, mayoristas, y generalistas en el sistema electoral uruguayo". Revista Uruguaya de Ciencia Politica.

Weingast, Barry, and William Marshall. 1988. “The industrial organization of Congress; or, Why legislatures like firms are not organized as markets". Journal of Political Economy 96:132-163.

\section{Documentos oficiales}

Constitución Política de la República Oriental del Uruguay (CPROU).

Reglamento Interno de la Cámara de Representantes de la República Oriental del Uruguay (RCR).

Reglamento Interno de la Cámara de Senadores de la República Oriental del Uruguay (RCS). 